\title{
Calcium to hydrogen line ratios in solar prominences
}

\author{
P. Gouttebroze ${ }^{1}$ and P. Heinzel ${ }^{2}$ \\ 1 Institut d'Astrophysique Spatiale, Univ. Paris XI/CNRS, Bât. 121, 91405 Orsay Cedex, France \\ 2 Astronomical Institute, Academy of Sciences of the Czech Republic, 25165, Ondřejov, Czech Republic \\ e-mail: pheinzel@asu.cas.cz
}

Received 8 January 2002 / Accepted 18 January 2002

\begin{abstract}
The ratio of Ca II $8542 \AA$ to $\mathrm{H} \beta$ line intensities has been used for a long time to diagnose the gas pressure in solar prominences. In this paper we reconsider the theoretical dependence of $E(8542) / E(\mathrm{H} \beta)$ on the gas pressure, as originally computed by Heasley \& Milkey (1978), and extend this theoretical correlation to higher pressures. Firstly, we revise the formation of calcium lines in prominences, using in parallel two independently developed NLTE radiative transfer codes. Computations consist of two subsequent steps: (i) the formation of hydrogen spectrum (treated in a similar way as in Gouttebroze et al. 1993), and (ii) the formation of calcium lines, using the electron-density structure obtained in step (i). The influence of hydrogen Lyman lines on Ca II to Ca III ionization is found to be very important for the determination of calcium-to-hydrogen line ratios. In particular, the intensities obtained for calcium lines at low pressures are significantly lower than those obtained by Heasley \& Milkey (1978), which is the result of a greater Ca III/Ca II ratio. Our numerical results have been further checked against an approximate analytical model. Secondly, we have performed an extended computation using a large grid of models covering different temperatures, gas pressures, geometrical thicknesses, microturbulent velocities and prominence altitudes. For temperatures lower than $10000 \mathrm{~K}$ and pressures lower than $0.1 \mathrm{dyn} \mathrm{cm}^{-2}$, the line ratio $E(8542) / E(\mathrm{H} \beta)$ undergoes only small variations, remaining between 0.2 and 0.3 . At higher pressures $(0.1$ to $1 \mathrm{dyn} \mathrm{cm}^{-2}$ ), the behaviour of this ratio appears to be strongly dependent on temperature: rapidly increasing below $6000 \mathrm{~K}$, moderately increasing between 6000 and $8000 \mathrm{~K}$, and generally decreasing at higher temperatures. A comparison of the present models with recent observations of Stellmacher \& Wiehr (2000) suggests the existence of cool prominence structures with temperatures around $6000 \mathrm{~K}$ and gas pressures higher than $0.1 \mathrm{dyn}^{\mathrm{cm}^{-2}}$.
\end{abstract}

Key words. sun prominences - radiative transfer - line: formation

\section{Introduction}

Several lines of hydrogen and ionized calcium are commonly observed in the optical spectra of solar prominences. For hydrogen, these observations concern principally the Balmer series, while the ionized calcium produces five lines of interest: the resonance lines $(\mathrm{H}$ and $\mathrm{K})$ and the infrared triplet. Since the formation conditions of hydrogen and calcium lines are different, some attempts were made to establish relations between the relative intensities of the lines from the two elements and the physical conditions in prominences, in order to interpret the observations.

Heasley \& Milkey (1978, hereafter HM78) compared the theoretical integrated intensities of two lines: one of the infrared lines of $\mathrm{Ca}$ II, at $8542 \AA$, and the $\mathrm{H} \beta$ line, at

Send offprint requests to: P. Gouttebroze, e-mail: goutte@ias.u-psud.fr
$4861 \AA$. They found that, in the temperature range [6500, $9000 \mathrm{~K}]$, the ratio of integrated intensities

$r=\frac{E(8542)}{E(H \beta)}$

was relatively insensitive to temperature $(T)$, practically independent of column mass, and regularly decreasing with gas pressure $(P)$. So, they derived a mean relation for $r(P)$ and subsequently used it to interpret observations. However, these computations of HM78 were restricted to 3 temperatures $(6500,7500$ and $9000 \mathrm{~K})$ and low pressures (about 0.003 to 0.2 dyn $\mathrm{cm}^{-2}$ ).

Gouttebroze et al. (1997, hereafter GVH97) investigated prominence models with higher pressures ( 0.1 to 1 dyn $\mathrm{cm}^{-2}$ ) and a wider range of temperatures (4300 to $15000 \mathrm{~K}$ ). They fixed the other parameters (slab thickness $2000 \mathrm{~km}$, microturbulent velocity $5 \mathrm{~km} \mathrm{~s}^{-1}$, altitude $10000 \mathrm{~km}$ ). As will be shown in the next section, these computations suffered from some inaccuracies, especially 
Table 1. Simultaneous observations of $E(8542)$ and $E(\mathrm{H} \beta)$.

\begin{tabular}{ll}
\hline Reference & $\begin{array}{l}E(8542) / E(\mathrm{H} \beta) \\
\text { (average value) }\end{array}$ \\
\hline Landman \& Illing (1977) & 0.375 \\
\hline de Boer et al. (1998) & 0.425 \\
\hline Stellmacher \& Wiehr (2000) & 0.63 (faint prominence) \\
& 0.28 (bright prominence) \\
\hline
\end{tabular}

concerning the ionization of calcium, but they produced new qualitative results which are confirmed by the present computations. In particular, they found that, at low temperatures, $r$ is greater for $P=1 \mathrm{dyn} \mathrm{cm}^{-2}$ than for $P=0.1 \mathrm{dyn} \mathrm{cm}^{-2}$. So, the mean slope of $r(P)$ in this range of pressures is positive, while it is negative at lower pressures, according to HM78. This is important for the diagnosis of prominences since, for a given value of $r$, we may have two different solutions for $P$, one in the low pressure range (given by the relation of HM78), and the other one at pressures higher than $0.1 \mathrm{dyn} \mathrm{cm}^{-2}$. For disk filaments, $8542 \AA$ line intensities were computed for a large grid of horizontal slab models (Tziotziou et al. 2001).

The two lines under consideration have been observed simultaneously in prominences by different authors. In Table 1, we present a (non exhaustive) list of such observations. The observed ratios $r$ vary approximately between 0.2 and 0.8 . If we apply the relation derived by HM78,

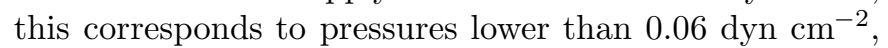
while GVH97 relations also predicts a solution at $P>$ 0.1 dyn $\mathrm{cm}^{-2}$.

Considering the importance of this relation for the determination of pressure, and the small number of models processed both by HM78 and GVH97, we present here an extended set of computations. In addition, comparative tests have been made between two independent codes in order to improve the accuracy of the computations, as compared to the previous ones reported by GVH97. These numerical methods are described in Sect. 2. The formation of lines is treated in Sect. 3, and illustrated by some numerical examples. In Sect. 4, we present the results obtained with a large set of models including the variations of many different parameters, and derive statistical relations. In the last section, we discuss the consequences of these results upon the interpretation of prominence observations.

\section{Numerical methods}

We use the same kind of models as in GVH97: a planeparallel slab standing vertically above the solar surface, and irradiated on both faces by the Sun. We solve the equations of radiative transfer, statistical equilibrium of level populations, and ionization equilibrium for the hydrogen atom. In this process, we obtain the radiation field inside the prominence as a function of depth and frequency. This radiation field is then used to compute the photoionization rates for the calcium atom. Photoionization of Ca II is driven by the internal radiation field of optically thick Lyman lines and Lyman continuum, and by UV continuum radiation longward of $912 \AA$ which is supposed to freely penetrate into the prominence slab. Then, the radiative transfer and statistical equilibrium equations are solved for the calcium atom, which yields level populations and line intensities. In this way, we obtain the synthetic spectra of hydrogen and calcium emitted by the prominence.

In order to optimize the accuracy of the computations, we used two independently developed codes, compared the results for several models, and improved these codes by mutual corrections.

The first code, developed by one of us (PG), is an improved version of that used by GVH97. Its hydrogen part is described in Gouttebroze \& Labrosse (2000). It is based on classical techniques, such as the Feautrier method with variable Eddington factors for solving the radiative transfer equations, and equivalent-two-level-atom (ETLA) technique for the statistical equilibrium equations. Partial frequency redistribution is taken into account for resonance lines of hydrogen and calcium. The model hydrogen atom includes 20 levels plus continuum, while the calcium atom includes one level for $\mathrm{Ca}$ I, five for Ca II and one for Ca III (it is exactly the same as in GVH97). The statistical equilibrium equations for determining the level populations are solved by ETLA iteration. The latest improvement consists of using a mixture of full and net radiative rates for the lines, instead of pure net rates. In this way, the number of iterations needed to achieve convergence in statistical equilibrium equations is reduced by a factor of 2 or 3 , depending on the model.

The other code, developed by $\mathrm{PH}$, is based on more modern techniques, such as multilevel accelerated Lambda iteration (MALI). The hydrogen part of this code is described in Heinzel (1995). The present version uses a hydrogen atom with 12 levels plus continuum and partial redistribution in Lyman lines. For Ca II-Ca III, it uses the same atomic model as the ETLA code.

The comparative procedure showed in particular the importance of Lyman lines for the Ca II to Ca III ionization. The head of the principal continuum is at $1044 \AA$, so that the corresponding photoionization rates are particularly sensitive to the intensities in the Lyman- $\beta$ line (1026 ̊) and, to a lesser extent, to subsequent Lyman lines (973 $\AA, 950 \AA$, etc.). Other continua (from higher levels) are also sensitive to the intensities in the Lyman$\alpha$ line $(1216 \AA)$. The code used by GVH97 was taking into account these lines, but the wavelength mesh used for interpolation was too coarse within the Lyman line profiles, so that the ionization rates were inaccurate, and the ratio (Ca III/Ca II) generally underestimated. The code written by $\mathrm{PH}$, using frequency-integrated intensities in the Lyman lines to compute the calcium photoionization rates, did not suffer from this interpolation problem. The PG code was then modified in order to use, in the photoionization rate computation, a variable-step wavelength mesh with high resolution $(0.01 \AA)$ in the cores of the Lyman lines, which ensures high accuracy. 
In addition, the intensities emitted by the solar surface, which are used (after dilution) as incident intensities on the faces of the prominence slab, have been revised. For eight Lyman lines ( $\mathrm{L} \beta$ to $\mathrm{L} \iota$ ), we presently use solar intensities measured by the SOHO/SUMER instrument (Warren et al. 1998). Consistently, we apply the full solution of radiative transfer equations to the nine first lines of the Lyman series, instead of four lines as in preceding papers (Gouttebroze et al. 1993, GVH97, Gouttebroze \& Labrosse 2000). For other lines, the incident intensities are the same as those used in GVH97.

After several minor corrections and modifications, the line intensities predicted by the two codes generally agree within $10 \%$. The results reported in the following sections are those finally obtained with the PG code only.

\section{Line formation}

In order to study the formation of hydrogen and calcium lines, we consider a small set of models with a temperature $T=7500 \mathrm{~K}$, which is the median temperature considered by HM78. The pressure $P$ varies from $10^{-3}$ to 1 dyn $\mathrm{cm}^{-2}$, with 7 points per decade. Concerning the thickness $D$ of the prominence, we adopt a relatively small value of $100 \mathrm{~km}$ (which corresponds to a prominence fine structure rather than to the whole prominence). In this way, we keep the $\mathrm{H} \beta$ and $8542 \AA$ lines optically thin for most pressures, which is more convenient for comparisons with analytical formulae. Slabs with larger sizes will be considered in the next section. Other parameters are fixed to mean values: the microturbulent velocity $\xi$ to $5 \mathrm{~km} \mathrm{~s}^{-1}$, and the altitude $H$ to $10000 \mathrm{~km}$.

\subsection{Hydrogen}

One particularity of the hydrogen atom is that all the excited levels are closer to the continuum than to the ground level, and that the distance between an excited level and the continuum decreases rapidly with the level number. One of the consequences is that the ratio $\left(n_{j} / n_{\mathrm{c}}\right)$, where $n_{j}$ is the number density of atoms in level $j$ and $n_{\mathrm{c}}$ that of protons, tends rapidly to its LTE value when $j$ increases. For the temperature of $7500 \mathrm{~K}$ and the different pressures, we have computed the mean populations of levels:

$N_{j}=\frac{1}{D} \int_{0}^{D} n_{j}(z) \mathrm{d} z$

and the mean LTE departure coefficients:

$b_{j}=\frac{N_{j}}{N_{\mathrm{c}}}\left(\frac{N_{\mathrm{c}}}{N_{j}}\right)_{\mathrm{LTE}}=\frac{N_{j}}{N_{\mathrm{c}}} \frac{1}{N_{\mathrm{e}} \Phi_{j \mathrm{c}}(T)}$.

where $N_{\mathrm{e}}$ is the average electron density from the model and

$\Phi_{j \mathrm{c}}(T)=\frac{g_{j}}{2 g_{\mathrm{c}}}\left(\frac{h^{2}}{2 \pi m_{\mathrm{e}} k T}\right)^{3 / 2} \mathrm{e}^{h \nu_{j c} / k T}$

(symbols have their usual meaning).

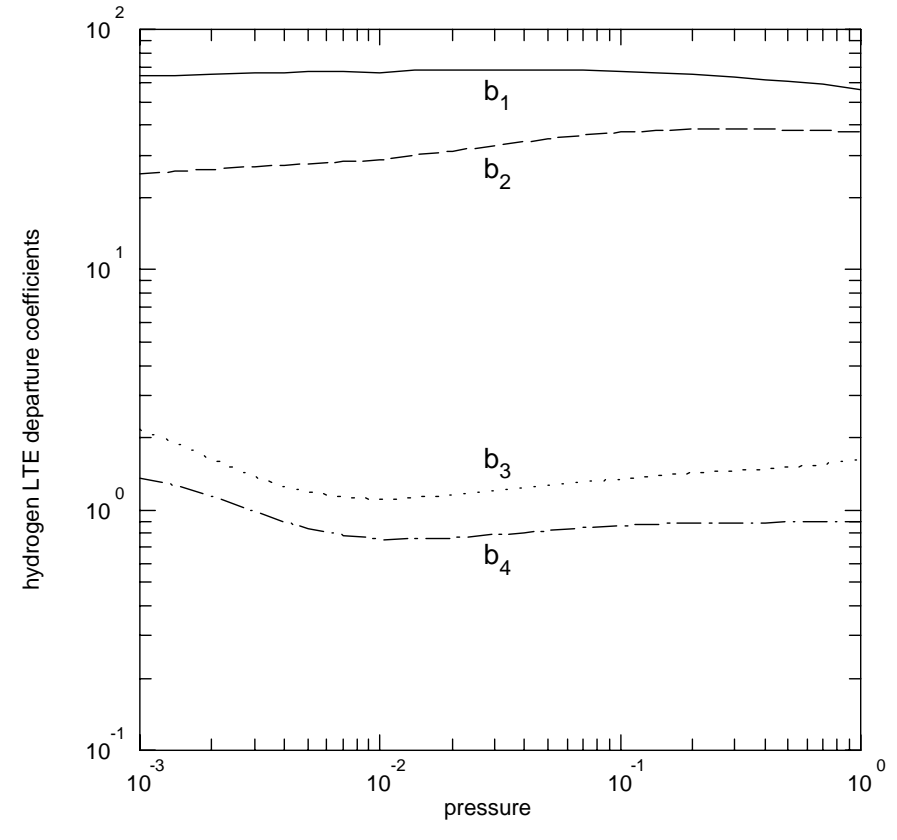

Fig. 1. Mean LTE departure coefficients $b_{j}$ for the 4 first levels of hydrogen, as a function of the gas pressure $\left(\mathrm{dyn} \mathrm{cm}^{-2}\right)$ in the slab.

The $b_{j}$ are represented in Fig. 1 , as a function of pressure, for levels 1 to 4 . For the ground level, the departure coefficient is almost constant and close to 60 , with a slight decrease at high pressures. For level 3, it varies between 1 and 2 and, for level 4, between 0.7 and 1.2. For higher levels, the departure coefficients are not represented, but they are still closer to 1 . Once $b_{1}$ is fixed, it is possible to evaluate approximately the electron density by assuming that hydrogen is the only electron contributor, so that $N_{\mathrm{e}}=N_{\mathrm{c}}$. With a helium-to-hydrogen number ratio of 0.1 , the state equation may be written as

$P=\left(1.1 N_{\mathrm{H}}+N_{\mathrm{e}}\right) k T$,

where $N_{\mathrm{H}}$ is the total hydrogen density. The populations of hydrogen excited levels being usually negligible, we have:

$N_{\mathrm{H}}=N_{1}+N_{\mathrm{e}}$.

From (3), we obtain:

$N_{1}=b_{1} N_{\mathrm{e}}^{2} \Phi_{1 \mathrm{c}}(T)$

which, combined with (5) and (6), gives the following equation for $N_{\mathrm{e}}$ :

$1.1 b_{1} \Phi_{1 \mathrm{c}}(T) N_{\mathrm{e}}^{2}+2.1 N_{\mathrm{e}}=\frac{P}{k T}$.

Its solution is:

$N_{\mathrm{e}}=N_{0}\left(\sqrt{\frac{P}{P_{0}}+1}-1\right)$

with

$N_{0}=\frac{2.1}{2.2 b_{1} \Phi_{1 \mathrm{c}}(T)}$ 
and

$P_{0}=1.05 N_{0} k T$.

Numerically, if $b_{1}=60$, we have $N_{0}=1.8 \times 10^{10} \mathrm{~cm}^{-3}$ and $P_{0}=0.020$ dyn $\mathrm{cm}^{-2}$.

The asymptotic values of expression (9) correspond to two different regimes: at low pressures $\left(P \ll P_{0}\right)$, hydrogen is mainly ionized and the electron density is roughly proportional to the pressure. For $P \gg P_{0}$, hydrogen is essentially neutral and $N_{\mathrm{e}}$ varies as the square root of $P$.

The intensity emitted in the optically thin $\mathrm{H} \beta$ line directly depends on the emission coefficient and consequently on the population of level 4 . The intensity emitted at frequency $\nu$ is

$I_{\nu}=e_{\nu} D$

with:

$e_{\nu}=\frac{h \nu_{24}}{4 \pi} N_{4} A_{42} \phi_{\nu}$,

$A_{42}$ being the spontaneous emission probability and $\phi_{\nu}$ the normalized emission profile. By integration over frequency, we obtain the total intensity emitted in $\mathrm{H} \beta$ by the slab:

$E(\mathrm{H} \beta)=\frac{h \nu_{24}}{4 \pi} N_{4} A_{42} D$.

One further approximation consists of assuming that the LTE departure coefficient $b_{4}$ is equal to 1 , which yields the following relation between the population of level 4 and the electron density:

$N_{4} \approx N_{\mathrm{e}}^{2} \Phi_{4 \mathrm{c}}(T)$.

Finally, we obtain:

$E(\mathrm{H} \beta) \approx \frac{h \nu_{24}}{4 \pi} A_{42} D \Phi_{4 \mathrm{c}}(T) N_{\mathrm{e}}^{2}$.

Numerically, with $\nu_{24}=6.17 \times 10^{14} \mathrm{~Hz}, A_{42}=8.40 \times$ $10^{6} \mathrm{~s}^{-1}, D=100 \mathrm{~km}, \Phi_{4 \mathrm{c}}(7500 \mathrm{~K})=3.8 \times 10^{-20}$, we have:

$E(\mathrm{H} \beta) \approx 10^{-18} N_{\mathrm{e}}^{2}$.

Combining (9) and (16), we obtain an approximation for computing the $\mathrm{H} \beta$ intensity as a function of the gas pressure. In Fig. 2, we compare this approximation with the intensities obtained from the detailed solution of radiative transfer and statistical equilibrium equations. The two values agree within $30 \%$.

The Eq. (16) indicates that the total intensity in the $\mathrm{H} \beta$ line is roughly proportional to the square of the electron density, and consequently to the emission measure $\int N_{\mathrm{e}}^{2} \mathrm{~d} z$. The same property was noticed for the $\mathrm{H} \alpha$ line in our previous papers (Gouttebroze et al. 1993; Heinzel et al. 1994). This property holds for all hydrogen lines, provided that their upper level LTE departure coefficient is close to 1 (or does not vary significantly with $P$ and $T$ ), and that they are optically thin (or marginally thick). In prominences, it is the case of almost all hydrogen lines, except those of the Lyman series.

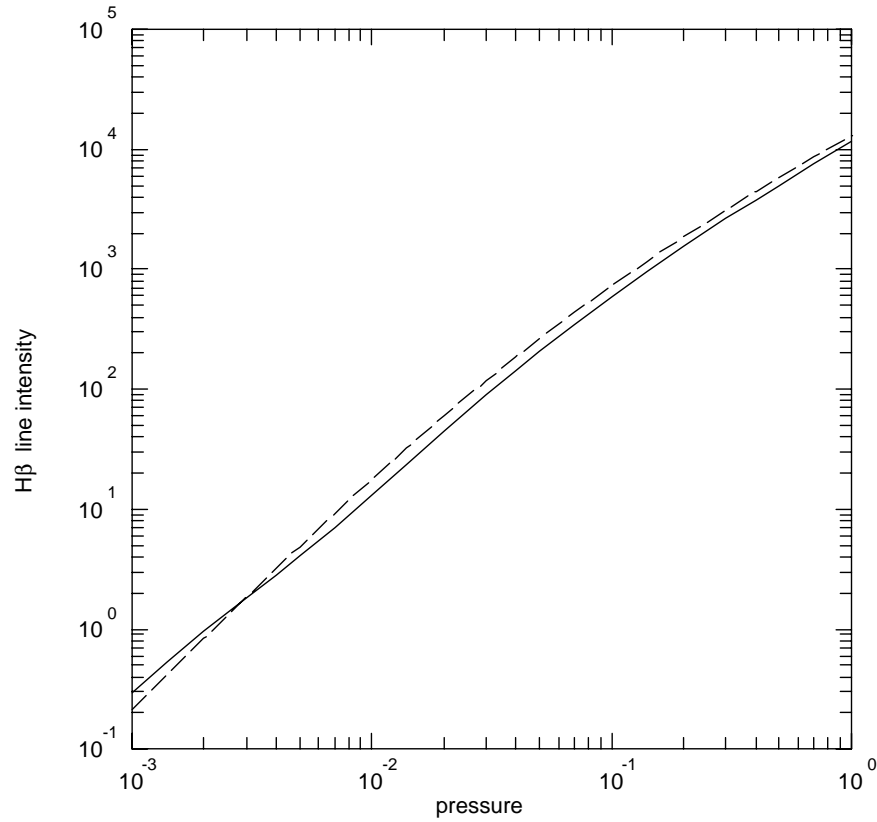

Fig. 2. Comparison of $\mathrm{H} \beta$ total intensities emitted by the slab (erg $\mathrm{cm}^{-2} \mathrm{~s}^{-1} \mathrm{sr}^{-1}$ ), as a function of pressure $\left(\mathrm{dyn} \mathrm{cm}^{-2}\right)$. Solid line: detailed computations; dashed line: approximation.

\subsection{Calcium}

As long as the $8542 \AA$ line is optically thin, which is the case for most models, its integrated intensity is proportional to the population of the upper level of the transition, which is the fifth level of Ca II. Thus, we derive for this line a relation similar to (14) for $\mathrm{H} \beta$ :

$E(8542) \approx \frac{h \nu_{35}}{4 \pi} N_{5}(\mathrm{Ca} \mathrm{II}) A_{53} D$.

This population of level 5 may be expanded as follows:

$N_{5}(\mathrm{Ca} I \mathrm{II})=\frac{N_{5}(\mathrm{Ca} \mathrm{II})}{N(\mathrm{Ca} \mathrm{II})} \times \frac{N(\mathrm{Ca} \mathrm{II})}{N(\mathrm{Ca})} \times \frac{N(\mathrm{Ca})}{N_{\mathrm{H}}} \times N_{\mathrm{H}}$.

The term $N(\mathrm{Ca}) / N_{\mathrm{H}}$ represents the abundance of calcium with respect to hydrogen. It is taken as constant and equal to $2.19 \times 10^{-6}$, as in GVH97 (HM78 use a very similar value: $\left.2.14 \times 10^{-6}\right)$.

The term $N(\mathrm{Ca} \mathrm{II}) / N(\mathrm{Ca})$ is the part of singly ionized calcium relative to the total population of calcium. For the models under investigation, the proportion of neutral calcium is negligible. On the contrary, the determination of the ionization equilibrium between Ca II and Ca III is a critical point, and the inclusion of Lyman line radiation field in the photoionization rates may change drastically this equilibrium. Figure 3 shows the mean calcium populations obtained with our test models, with and without the inclusion of Lyman lines. When the Lyman lines are included (Fig. 3a), the population of Ca III is larger than that of Ca II for pressures lower than $0.08 \mathrm{dyn}^{-2}$. For very low pressures, the population of Ca III may be 10 times larger than that of Ca II. On the contrary, when the Lyman lines are neglected (Fig. 3b), Ca II populations dominate everywhere, except at very low pressures $\left(P<0.003\right.$ dyn $\left.\mathrm{cm}^{-2}\right)$. 


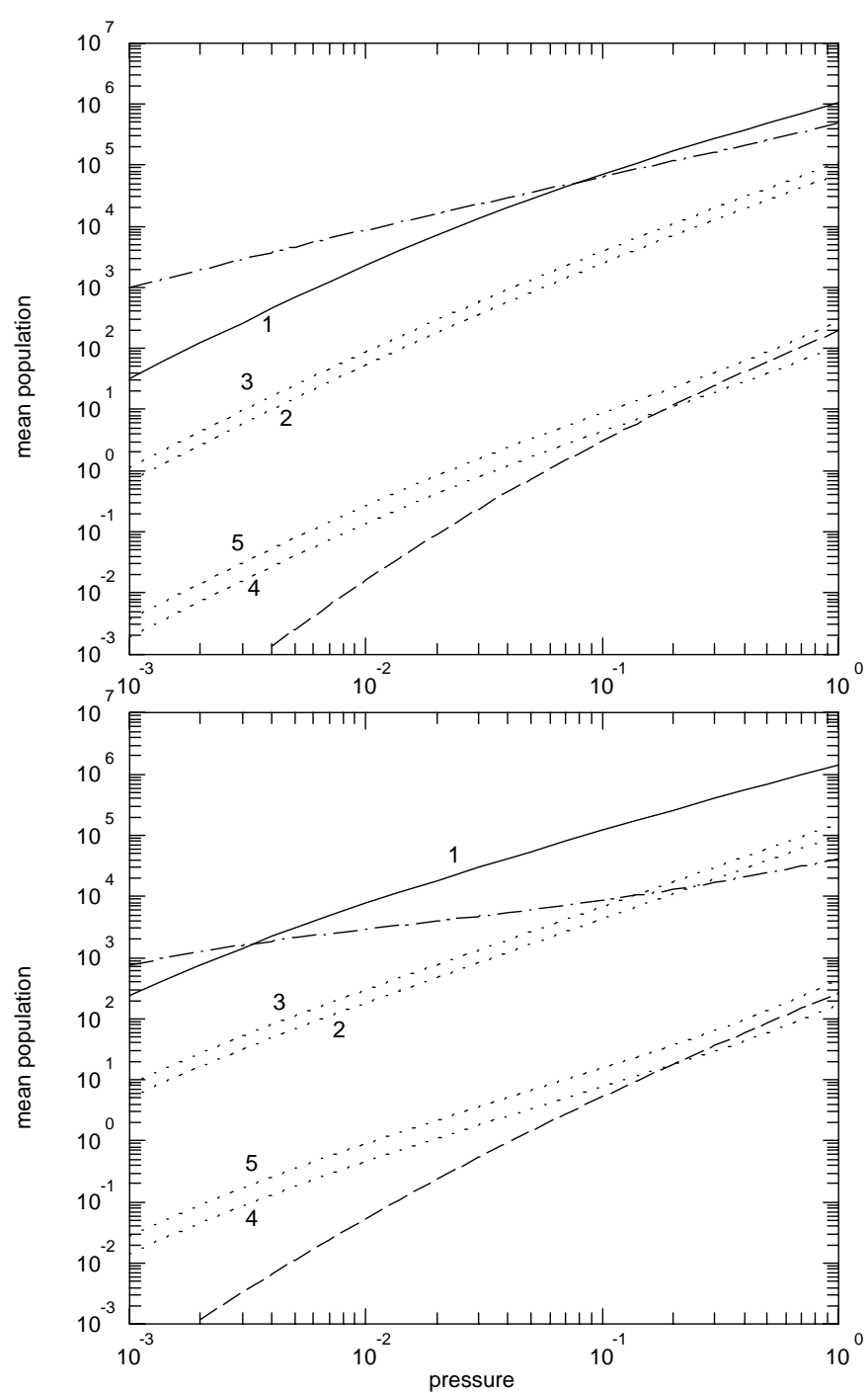

Fig. 3. Mean calcium level populations as a function of the gas pressure $\left(\right.$ dyn $\left.\mathrm{cm}^{-2}\right)$ in the slab. Ca I: dashed line. Ca II, ground level: solid line. Ca II, excited levels: dotted lines. Ca III: dash-dotted line. Ca II levels are labelled by order of increasing energy. a) with Lyman lines included in photoionization rates (upper panel). b) without Lyman lines (lower panel).

The term $N_{5}$ (Ca II) $/ N(\mathrm{Ca}$ II) is the ratio of the population of the fifth level compared to the total population of Ca II, which is practically equal to that of the ground state. In Fig. 3a, the curves corresponding to the different levels of Ca II seem to be approximately parallel, which means that the ratio $N_{5} / N_{1}$ does not vary very much. This variation is detailed in Fig. 4, which shows that this ratio is effectively constant between 0.001 and 0.1 dyn $\mathrm{cm}^{-2}$, but begins to rise at higher pressures. On this figure, we also show the ratio corresponding to LTE, which is a function of temperature $\left(1.53 \times 10^{-2}\right.$ for $\left.7500 \mathrm{~K}\right)$, and the "optically thin ratio", obtained by solving the statistical equilibrium equations with intensities defined by the incident radiation, and neglecting collisions. This last ratio, which is independent of temperature, is equal to $1.16 \times 10^{-4}$ in the present case. Thus Fig. 4 shows that, at low pressures,

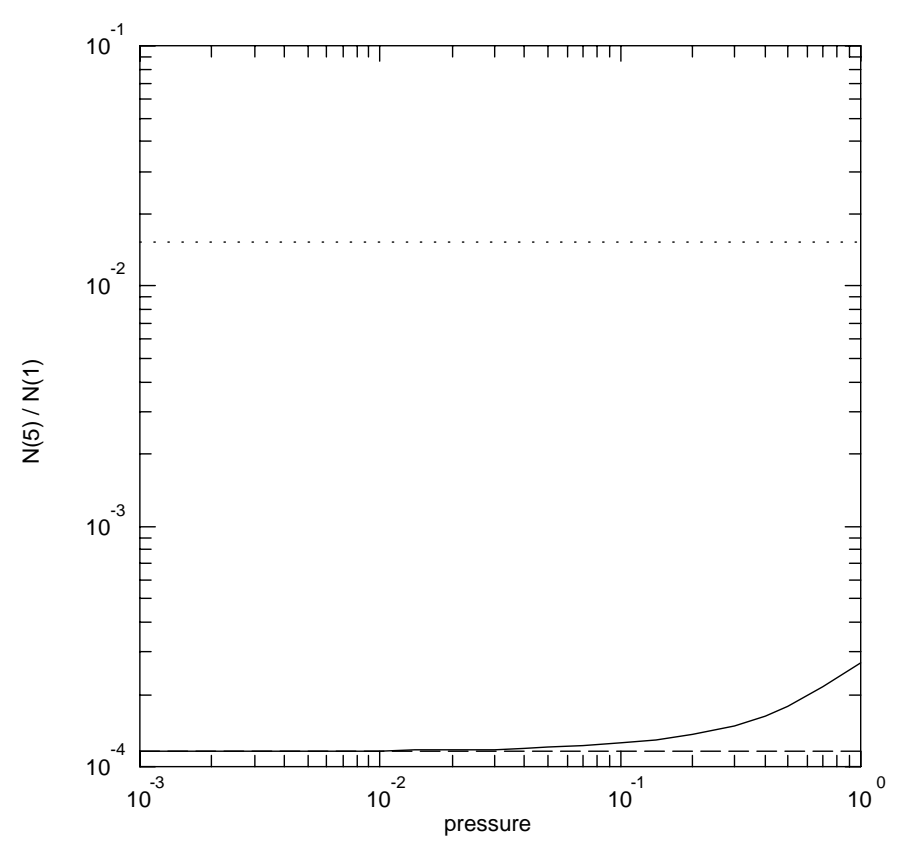

Fig. 4. Population ratio $\left(N_{5} / N_{1}\right)$ for Ca II as a function of pressure. Solid line: result of numerical computations. Dashed line: optically thin limit. Dotted line: value corresponding to LTE.

$N_{5} / N_{1}$ remains nearly equal to its optically thin value, and begins to rise at high pressures to get closer to its LTE value, when collisions cease to be negligible.

In the case where all calcium atoms are singly ionized, i.e. where $N(\mathrm{Ca} \mathrm{II}) / N(\mathrm{Ca})=1$, we can derive a simple approximation for the intensity in the $8542 \AA$ line. To this purpose, we substitute in $(19) N_{5}(\mathrm{Ca}$ II $) / N(\mathrm{Ca}$ II $)$ with its optically thin limit $1.16 \times 10^{-4}$ and $N(\mathrm{Ca}) / N_{\mathrm{H}}$ with $2.19 \times 10^{-6}$. We obtain in this way:

$N_{5}(\mathrm{Ca}$ II $) \approx 2.5 \times 10^{-10} N_{\mathrm{H}}$

and, with the help of (18):

$E(8542) \approx 3.4 \times 10^{-9} N_{\mathrm{H}}$.

If we combine this equation with the approximation (17) for $\mathrm{H} \beta$, we have:

$r=\frac{E(8542)}{E(\mathrm{H} \beta)} \approx 3.4 \times 10^{9} \frac{N_{\mathrm{H}}}{N_{\mathrm{e}}^{2}}$.

Since $N_{\mathrm{e}}^{2}$ increases more rapidly with pressure than $N_{\mathrm{H}}$, the approximation (22) corresponds to a decrease of $r(P)$. However, this relation is not really useful in practice because, at the same time, the ionization ratio (Ca III/Ca II) decreases with pressure, producing a non-monotonic variation of $r(P)$. This is illustrated in Fig. 5, where we compare the approximation (22), the relation obtained by HM78, and the two present numerical results, one obtained in taking into account the ionizing radiation of Lyman lines, and the other in neglecting it. The approximation (22) and the relation of HM78 are continuously decreasing. The relation obtained in neglecting Lyman lines 


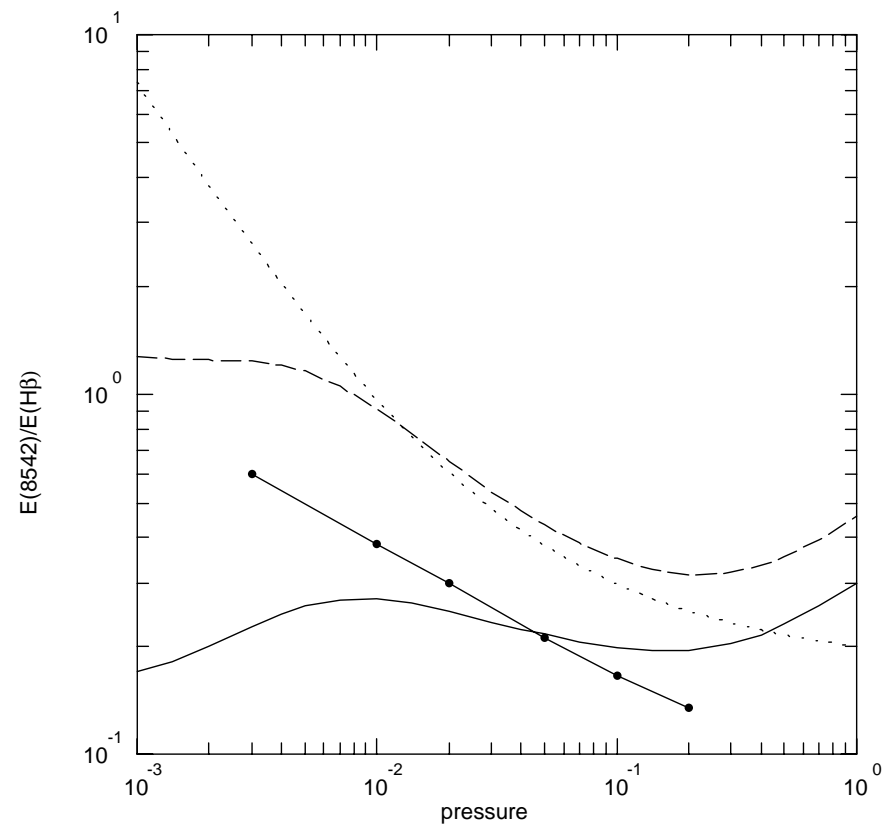

Fig. 5. Ratio of line intensities $r=E(8542) / E(\mathrm{H} \beta)$, as a function of pressure. Solid line: present computations, with Lyman lines included in photoionization rates. Dashed line: same computations with Lyman lines omitted. Solid line plus dots: results of Heasley \& Milkey (1978). Dotted line: approximation defined by Eq. (22).

is generally decreasing, except at high pressures. The relation obtained with full Lyman line radiation is relatively flat with a maximum near 0.008 and a minimum near 0.2 dyn $\mathrm{cm}^{-2}$. The difference between our two calculations may be understood from Fig. 3: on (3b), Ca II dominates Ca III for $P>0.003$ while, on (3a), it is true only for $P>0.2$.

\section{Statistical study}

In order to investigate the diagnostic value of the ratio $r$, it is necessary to study the intensity variations of the $\mathrm{H} \beta$ and $8542 \AA$ lines when all relevant parameters vary simultaneously. In the past (Gouttebroze et al. 1993), we used a grid of models with fixed values for the different parameters. However, the number of models required by such a procedure increases rapidly with the number of parameters and, for this reason, we allowed only variations for 3 parameters (temperature, pressure and thickness) and fixed the microturbulent velocity and the altitude to constant values. In the present study, we use a different approach: we attribute to each parameter $(T, P, D, \xi$ and $H)$ a range of variation and, for each model, we choose randomly the parameter value within the corresponding range of variation. These ranges of variation of the parameters are indicated in Table 2 . Within each range, the probability density is taken as constant with respect to the logarithm of the parameter (for instance, there are statistically as many models with $T$ between 5000 and $6000 \mathrm{~K}$ as between 15000 and $18000 \mathrm{~K}$ ). With this procedure, the number of models becomes rather arbitrary. In addition,

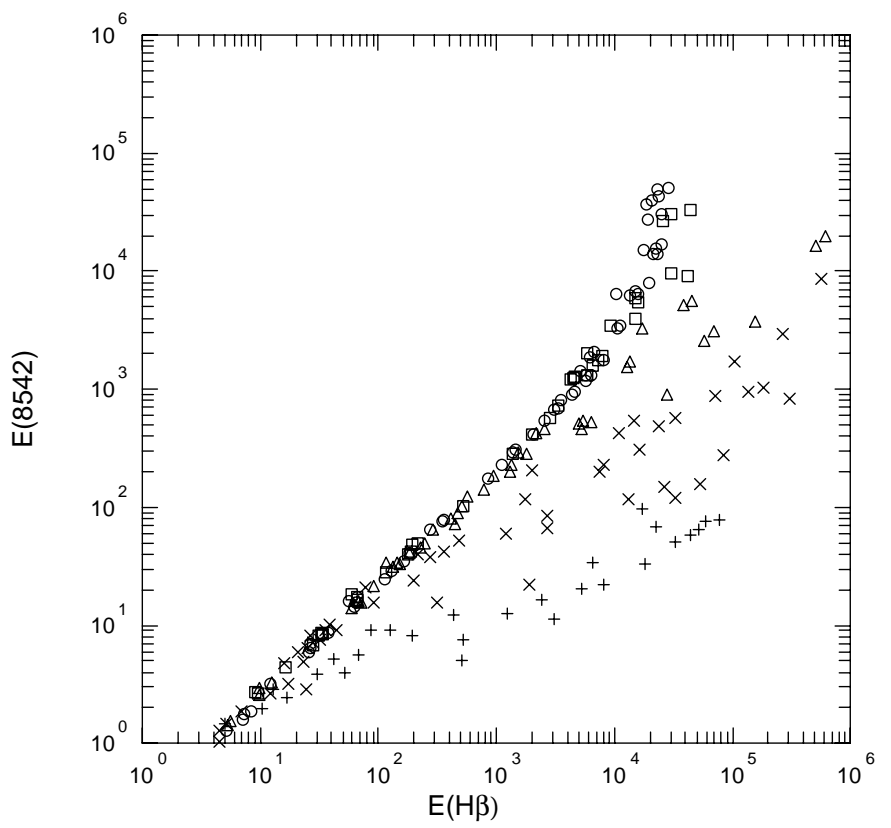

Fig. 6. Intensity in the Ca II $8542 \AA$ line vs. intensity in the $\mathrm{H} \beta$ line, for 250 prominence models with random parameters. Symbols indicate the temperature of the model: circles: 4000 to $6000 \mathrm{~K}$; squares: 6000 to $8000 \mathrm{~K}$; triangles: 8000 to $10000 \mathrm{~K}$; "×": 10000 to $15000 \mathrm{~K}$; "+": 15000 to $20000 \mathrm{~K}$.

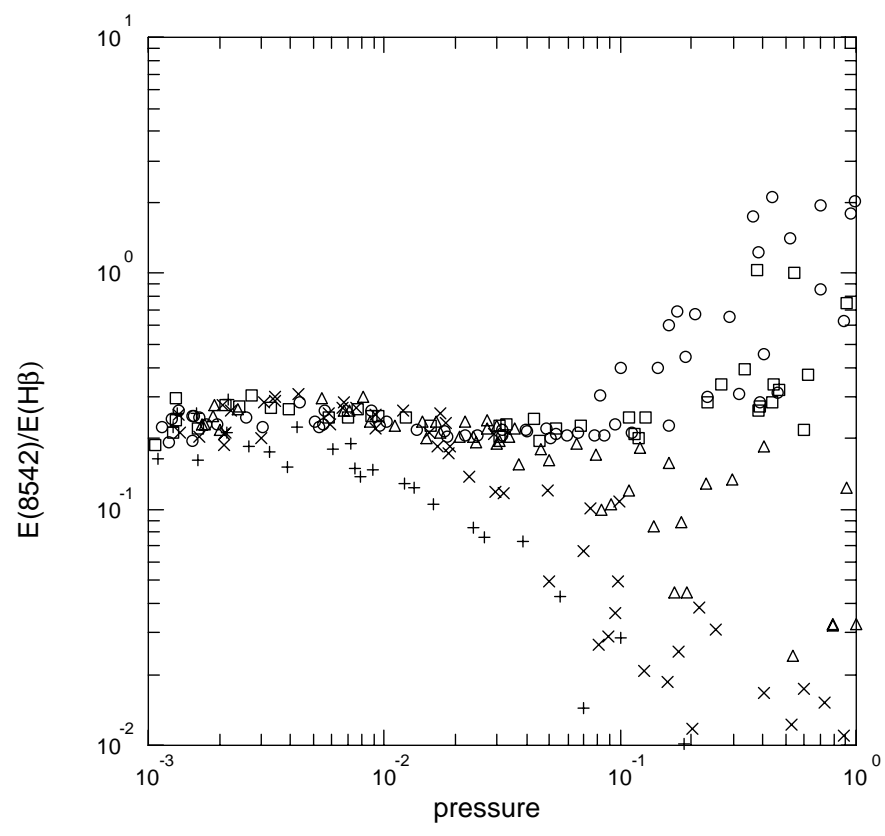

Fig. 7. Line intensity ratio $r$ as a function of pressure $\left(\right.$ dyn $\left.\mathrm{cm}^{-2}\right)$. Symbols indicate temperature as in Fig. 6 .

the set of models so obtained bears more resemblance to observations than a grid of models with regularly spaced parameter values.

In Fig. 6, we have represented the intensity emitted in the calcium infrared line as a function of that in the $\mathrm{H} \beta$ line. For clarity, the number of models has been limited to 250 , and the temperatures are indicated by different symbols. It appears that, if we exclude the models with 
Table 2. Ranges of variation for model parameters.

\begin{tabular}{lllll}
\hline quantity & symbol & unit & minimum & maximum \\
\hline temperature & $T$ & $\mathrm{~K}$ & 4000 & 20000 \\
gas pressure & $P$ & $\mathrm{dyn} \mathrm{cm}{ }^{-2}$ & 0.001 & 1 \\
thickness & $D$ & $\mathrm{~km}$ & 100 & 5000 \\
microturbulent velocity & $\xi$ & $\mathrm{km} \mathrm{s}^{-1}$ & 2 & 8 \\
altitude & $H$ & $\mathrm{~km}$ & 3000 & 30000 \\
\hline
\end{tabular}

a temperature larger than $10000 \mathrm{~K}$ (represented by " $\times$ " or "+"), there is a very good correlation between the intensities of the two lines for low or moderate intensities. This means that, at moderate temperatures or pressures, the ratio of the two lines is practically independent of the other three parameters. At temperatures higher than $10000 \mathrm{~K}$, the intensity of the calcium line decreases with respect to that of the hydrogen line. These conclusions are confirmed by Fig. 7, which represents the ratio $r$ as a function of pressure. For temperatures lower than $10000 \mathrm{~K}$

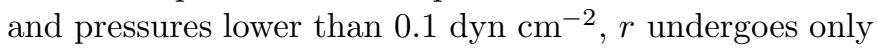
small variations, and generally remains between 0.2 and

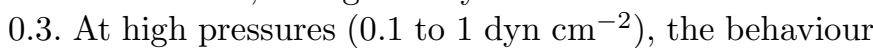
of $r$ appears to be strongly dependent on temperature: rapidly increasing below $6000 \mathrm{~K}$, moderately increasing between 6000 and $8000 \mathrm{~K}$, and generally decreasing at higher temperatures.

In order to quantify this relation with pressure and temperature, we computed line intensities for a larger set of about 4000 models and, for different ranges of temperatures, we used a least square technique to derive approximate relations for $r(P)$. This procedure is illustrated in Fig. 8, for the temperature range $[6000,8000 \mathrm{~K}]$. The fit function is a polynomial of the fourth degree:

$\log (r)=\sum_{i=1}^{5} a_{i}[\log (P)]^{i-1}$.

The use of polynomials of higher degrees does not produce any significant decrease of the standard deviation. Figure 8 also shows the dispersion of the results, which is small below $0.1 \mathrm{dyn} \mathrm{cm}^{-2}$, and increases rapidly above. This is due to the fact that, at low pressures, the intensities are roughly proportional to the thickness of the slab while, at high pressures, their dependence on $D$ becomes nonlinear. The same operation was performed for 5 different ranges of temperature, and the results are displayed in Fig. 9, which confirms the existence of a change of trend of $r(P)$ around a temperature of $8000 \mathrm{~K}$. At lower temperatures, $r(P)$ is increasing at high pressures, while at higher temperatures it is decreasing. The coefficients $a_{i}$ of Eq. (23) are given in Table 3.

\section{Discussion and conclusion}

There is another parameter which was not discussed in the preceding sections: the abundance of calcium. Its effect on the value of $r$ is simple, as long as the two lines are optically thin, which is usually the case: the intensity of

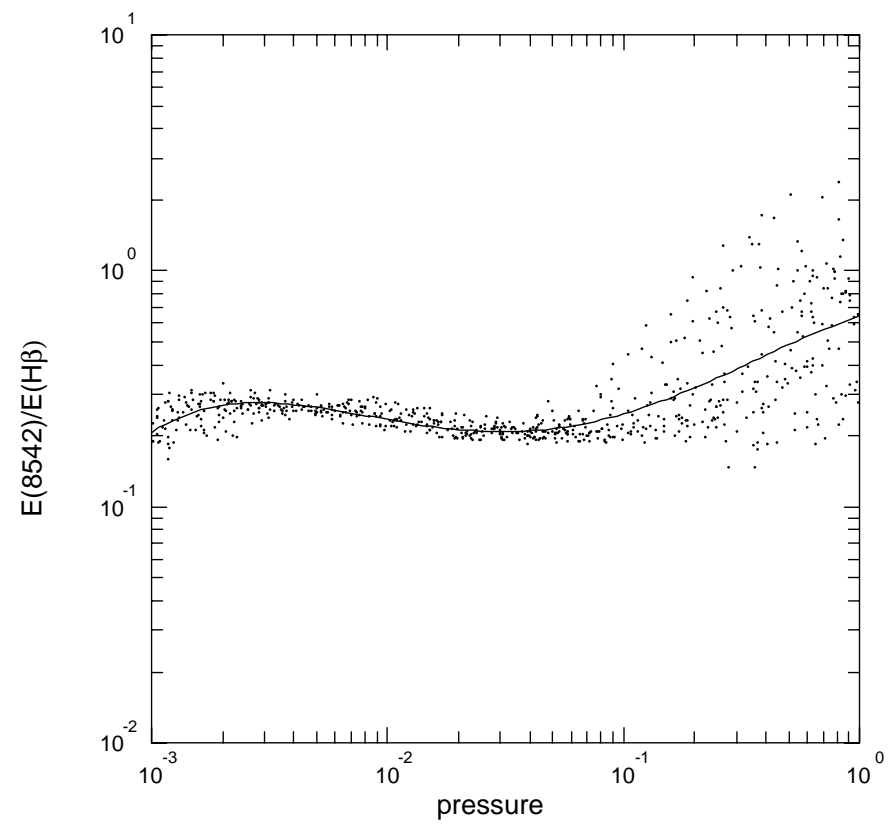

Fig. 8. Least square determination of the relation $r(P)$ for the temperature range $[6000,8000 \mathrm{~K}]$. Each point corresponds to one model. The least square approximation (solid line) is obtained using a fourth degree polynomial.

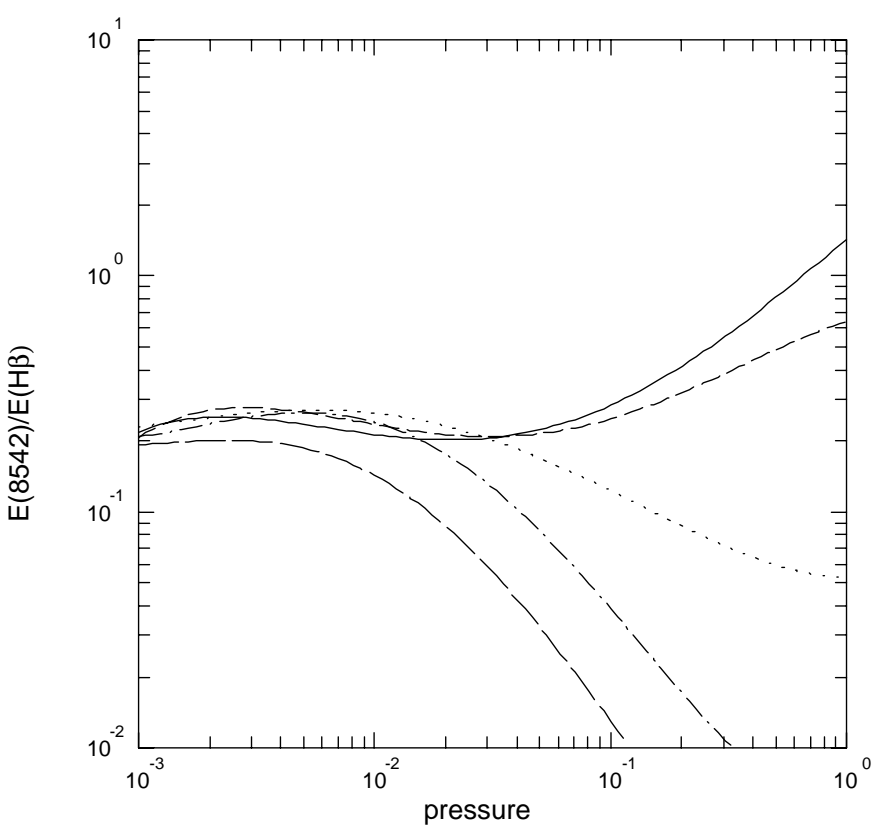

Fig. 9. Least square fits of $r(P)$ for different temperature ranges: solid line: 4000 to $6000 \mathrm{~K}$; dashed line: 6000 to $8000 \mathrm{~K}$; dotted line: 8000 to $10000 \mathrm{~K}$; dash-dots: 10000 to $15000 \mathrm{~K}$; long dashes: 15000 to $20000 \mathrm{~K}$. 
Table 3. Coefficients of polynomial (23) for 5 different temperature ranges $\left[T_{\min }, T_{\max }\right]$.

\begin{tabular}{lllllll}
\hline$T_{\min }$ & $T_{\max }$ & $a_{1}$ & $a_{2}$ & $a_{3}$ & $a_{4}$ & $a_{5}$ \\
\hline 4000 & 6000 & 0.1552 & 0.8131 & -0.0885 & -0.2532 & -0.0545 \\
6000 & 8000 & -0.1914 & 0.3138 & -0.3913 & -0.3639 & -0.0723 \\
8000 & 10000 & -1.2783 & -0.0229 & 0.6117 & 0.3080 & 0.0417 \\
10000 & 15000 & -2.2918 & -0.0574 & 1.4673 & 0.7451 & 0.1031 \\
15000 & 20000 & -2.8906 & -0.0851 & 1.5691 & 0.7466 & 0.0982 \\
\hline
\end{tabular}

the $8542 \AA$ line is proportional to the ratio $(\mathrm{Ca} / \mathrm{H})$, while that of the $\mathrm{H} \beta$ line is independent of this ratio. As a consequence, $r$ is practically proportional to $(\mathrm{Ca} / \mathrm{H})$, except for a few models with high values of $P$ and $D$.

So, as long as $(\mathrm{Ca} / \mathrm{H})$ remains close to $2.2 \times 10^{-6}$, the ratio $r$ remains in the range $[0.2,0.3]$ for low or moderate pressures $\left(P<0.1\right.$ dyn $\left.\mathrm{cm}^{-2}\right)$. At higher pressures, $r(P)$ increases if the temperature is lower than $8000 \mathrm{~K}$, and decreases for higher temperatures. We can compare these results with the observations quoted in Table 1 . In particular, Stellmacher \& Wiehr (2000) observed two prominences, one bright and one weak. Concerning the bright prominence, they obtained values of $r$ between 0.15 and 0.40 , with a maximum of occurence near 0.28 . In this case, our calculations have little diagnostic value since, as may be seen in Fig. 7, models with any pressure may produce such values of $r$. On the contrary, the faint prominence observed by the same authors is characterized by higher values of $r$, in the range $[0.5,0.8]$. Such values are found at pressures larger than $0.1 \mathrm{dyn} \mathrm{cm}^{-2}$ and temperatures lower than $8000 \mathrm{~K}$ (and principally, lower than $6000 \mathrm{~K}$ ). This would suggest the existence of cool and relatively dense cores in this object. In this respect, it is interesting to note that Stellmacher \& Wiehr (2000) report a relative constancy of $r$ over whole prominences. With our results for $r \approx[0.2,0.3]$, this means that their bright prominence can have internal pressure variations in the range, say, $P \approx\left[10^{-3}, 10^{-1} \mathrm{dyn}_{\mathrm{cm}^{-2}}\right]$. But for the fainter prominence, it is rather difficult to understand the constancy of $r$. Therefore, new observations are required to check this behaviour. It is also interesting to note that, in prominence models based on magnetohydrostatic equilibrium (such as those of the Kippenhahn-Schlüter type), the pressure in the core of the prominence is much higher than in the external medium (see Heinzel \& Anzer 2001).

Finally, there remain certain uncertainties concerning the interpretation of the relatively high values of $r(>0.5)$ observed in some prominences. This may be the signature of cold and dense structures, but might as well be the result of a local calcium abundance higher than expected. Other mechanisms, like e.g. an increased incident radiation from a surrounding plage, seem to reduce $r$ to even lower values. This illustrates the fragility of diagnostics based on the intensities in two lines only. The present computations produce not only integrated intensities in the Lyman, Balmer, Paschen lines of hydrogen and infrared and resonance lines of Ca II, but also the profiles of these different lines. So, the future of prominence diagnostic is certainly based on multi-line comparisons between computations and observations and, when the spectral resolution is sufficient, on the use of line profiles.

Acknowledgements. We are indebted to Harry Warren for providing us with Lyman line observations from SUMER. We also wish to thank Ulrich Anzer, Jean-Claude Vial and Nicolas Labrosse for critical reading of the manuscript, and the referee for his valuable comments. The computations were performed at IDRIS (Institut du Développement et des Ressources en Informatique Scientifique) and MEDOC (Multi-Experiment Data Operation Centre for SOHO). PG acknowledges financial support and hospitality from the Ondřejov Observatory during his visit in August 2001. PH was supported by CNRS and by the grant A3003902 of the Academy of Sciences of the Czech Republic.

\section{References}

de Boer, C. R., Stellmacher, G., \& Wiehr, E. 1998, A\&A, 334, 280

Gouttebroze, P., Heinzel, P., \& Vial, J.-C. 1993, A\&AS, 99, 513

Gouttebroze, P., \& Labrosse, N. 2000, Solar Phys., 196, 349

Gouttebroze, P., Vial, J.-C., \& Heinzel, P. 1997, Solar Phys., 172, 125 (GVH97)

Heasley, J. N., \& Milkey, R. W. 1978, ApJ, 221, 677 (HM78)

Heinzel, P. 1995, A\&A, 299, 563

Heinzel, P. \& Anzer, U. 2001, A\&A, 375, 1082

Heinzel, P., Gouttebroze, P., \& Vial, J.-C. 1994, A\&A, 292, 656

Landman, D. A., \& Illing, R. M. E. 1977, A\&A, 55, 103

Stellmacher, G., \& Wiehr, E. 2000, Solar Phys., 196, 357

Tziotziou, K., Heinzel, P., Mein, P., \& Mein, N. 2001, A\&A, 366, 686

Warren, H. P., Mariska, J. T., \& Wilhelm, K. 1998, ApJS, 119, 105 